\title{
STABLE ANALYSIS FOR A DELAYED POPULATION MODEL WITH SURVIVAL RATE
}

\author{
ZHI-QIANG ZHU
}

Received 22 September, 2018

\begin{abstract}
In this paper, we consider a discrete population model and obtain a couple of criteria to guarantee our model being stable, including the global attractor, the extreme stability, and the periodicity.
\end{abstract}

2010 Mathematics Subject Classification: 39A30; 39A23

Keywords: delayed population model, invariant set, extreme stability, periodic solution, fixed point theorem

\section{INTRODUCTION}

This paper is devoted to consider the stable behavior for a discrete population model with survival rate given by

$$
x(t+1)=\gamma(t) x(t)+x(\tau(t)) e^{r(t)\left(1-\frac{x(\tau(t))}{k(t)}\right)}, t \in \mathbb{Z}_{+},
$$

where $\mathbb{Z}_{+}$is the set of nonnegative integers, $\gamma(t) \in(0,1)$ is the survival rate for each $t \in \mathbb{Z}_{+}, \tau(t) \in \mathbb{Z}_{+}$denotes the delay in $t$, and $r(t)$ and $k(t)$ stand respectively, for the intrinsic growth rate and the carrying capacity of the habitat.

The motivation of our considerations stems from the papers [2, 3, 5, 6, 8]. Concretely speaking, Diagana in [2] studied the periodic problem of population models of the form

$$
x(t+1)=\gamma(t) x(t)+f(t, x(t)), \quad t \in \mathbb{Z},
$$

where $\mathbb{Z}$ is the set of integers. Hamaya considered the almost periodic solutions in [3] for the discrete Ricker delay model

$$
x(t+1)=x(t) e^{f(t, x(t-\tau(t)))}, t \in \mathbb{Z}_{+} .
$$

In $[5,8]$ Liz and Saker et al. discussed respectively, the qualitative behavior of periodic Ricker equation

$$
x(t+1)=x(t) e^{r(t)-x(t)}
$$

This research was supported by the NNSF of China (No. 11671406).

(c) 2019 Miskolc University Press 
and in [6], the authors investigated the extreme stability of the following discrete logistic equation

$$
x(t+1)=x(t) e^{r(t)\left(1-\frac{x(t)}{k(t)}\right)}, t \in \mathbb{Z}_{+} .
$$

We observe that the stability is of vital importance when considering the periodicity of a model, see, for example, [3, 6,9]. In this paper, we will concentrate our attention on the stability of (1.1), including the global attractor, the extremely stability and the periodicity.

In what follows, we always assume that

(H1) there exist $\gamma_{0}$ and $\gamma_{1}$ in $(0,1)$, and an integer $\tau_{0}>0$ such that

$$
\inf _{t \in \mathbb{Z}_{+}} \gamma(t)=\gamma_{0}, \sup _{t \in \mathbb{Z}_{+}} \gamma(t)=\gamma_{1}
$$

and

$$
t-\tau_{0} \leq \tau(t) \leq t \text { for } t \in \mathbb{Z}_{+} ;
$$

(H2) there exist $r_{i}$ and $k_{i}$ in $(0, \infty)$ for $i=0,1$, such that

$$
r_{0} \leq r(t) \leq r_{1} \text { and } k_{0} \leq k(t) \leq k_{1} \text { for } t \in \mathbb{Z}_{+},
$$

where

$$
\inf _{t \in \mathbb{Z}_{+}} r(t)=r_{0}, \sup _{t \in \mathbb{Z}_{+}} r(t)=r_{1}, \inf _{t \in \mathbb{Z}_{+}} k(t)=k_{0}, \quad \sup _{t \in \mathbb{Z}_{+}} r(t)=k_{1} ;
$$

(H3) the functions $L(x)$ and $U(x)$, and the constants $M$ and $m$ are defined, respectively, by

$$
L(x)=x e^{r_{0}-\frac{r_{1} x}{k_{0}}} \text { and } U(x)=x e^{r_{1}-\frac{r_{0} x}{k_{1}}} \text { for } x \geq 0
$$

as well as

$$
M=U\left(\frac{k_{1}}{r_{0}}\right) \text { and } m=L\left(\frac{M}{1-\gamma_{1}}\right) ;
$$

(H4) for $\delta \geq 1$, the constant $\Delta$ is defined by

$$
\Delta= \begin{cases}L\left(\delta M\left(1-\gamma_{1}\right)^{-1}\right)=\frac{\delta M}{1-\gamma_{1}} e^{r_{0}-\frac{\delta r_{1} M}{k_{0}\left(1-\gamma_{1}\right)}}, & L\left(\delta M\left(1-\gamma_{1}\right)^{-1}\right)<\frac{r_{0} k_{0}}{r_{1}}, \\ \frac{r_{0} k_{0}}{r_{1}}, & \text { otherwise, }\end{cases}
$$

where $\frac{r_{0} k_{0}}{r_{1}}$ is the fixed point of $L(x)$. See the following diagram when allowing $\delta=1$ and $\left(1-\gamma_{1}\right)^{-1}=1$ : 


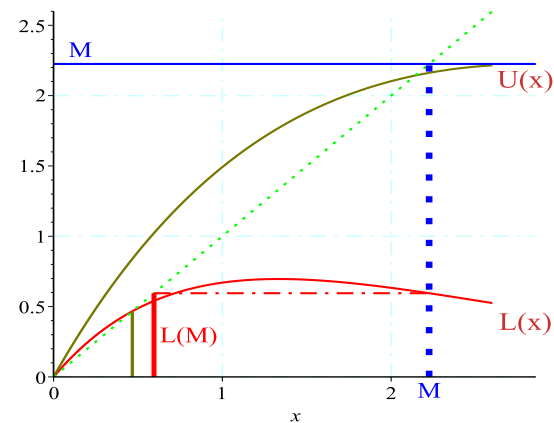

Let $\mathscr{D}\left[-\tau_{0}, 0\right]$ be the set of all discrete functions $\varphi:\left\{-\tau_{0},-\tau_{0}+1, \ldots, 0\right\} \rightarrow \mathbb{R}$ with the norm $|\varphi|=\max _{-} \tau_{0} \leq t \leq 0|\varphi(t)|$. For a given $\varphi \in \mathscr{D}\left[-\tau_{0}, 0\right]$, from (1.1) we can get a unique sequence $\{x(t)\}_{t \geq-\tau_{0}}$ satisfying $x(t)=\varphi(t)$ for $t=-\tau_{0},-\tau_{0}+1, \ldots, 0$. This sequence is called a solution of (1.1), and sometimes we denote this solution by $\{x(t ; \varphi)\}_{t \geq-\tau_{0}}$.

To continue our discussions in the sequel, we require some notions as follows.

Definition 1 ([9]). (i) The set $S \subset \mathbb{R}$ is said to be invariant relative to (1.1) if for $\varphi \in \mathscr{D}\left[-\tau_{0}, 0\right]$ with $\varphi(t) \in S$ for $t \in\left[-\tau_{0}, 0\right]$, the solution $\{x(t ; \varphi)\}_{t \geq-\tau_{0}}$ of (1.1) satisfies $x(t ; \varphi) \in \delta$ for all $t \geq-\tau_{0}$.

(ii) The set $S \subset \mathbb{R}$ is said to be a global attractor of (1.1) if for any $\varepsilon>0$ and positive function $\varphi \in \mathscr{D}\left[-\tau_{0}, 0\right]$, there exists an integer $N(\varepsilon, \varphi)>0$ such that the solution $\{x(t ; \varphi)\}_{t \geq-\tau_{0}}$ of (1.1) satisfies

$$
\min _{s \in S}|x(t ; \varphi)-s|<\varepsilon \text { for all } t \geq N(\varepsilon, \varphi) .
$$

Definition 2 ([6]). We say (1.1) to be extremely stable provided any two positive solutions $\{x(t)\}_{t \geq-\tau_{0}}$ and $\{y(t)\}_{t \geq-\tau_{0}}$ of (1.1) satisfy

$$
\lim _{t \rightarrow \infty}|x(t)-y(t)|=0 .
$$

Definition 3 ([7]). A sequence $\{x(t)\}_{t \geq-\tau_{0}}$ is said to be asymptotically $\sigma$-periodic if there exist two sequences $\{p(t)\}_{t \geq-\tau_{0}}$ and $\{q(t)\}_{t \geq-\tau_{0}}$ such that

$$
x(t)=p(t)+q(t),
$$

where $\{p(t)\}_{t \geq-\tau_{0}}$ is a $\sigma$-periodic sequence, and $q(t) \rightarrow 0$ as $t \rightarrow \infty$.

\section{Stability of (1.1)}

In this section we study the stability of (1.1). For this end, we first note that any solution $\{x(t ; \varphi)\}_{t \geq-\tau_{0}}$ of (1.1) verifies that

$$
x(t ; \varphi)=\prod_{v=0}^{t-1} \gamma(v) \varphi(0)+\sum_{v=0}^{t-1}\left[\prod_{s=v}^{t-2} \gamma(s+1)\right] f(v, x(\tau(v))), t \geq 1,
$$


where

$$
f(v, u)=u e^{r(v)\left(1-\frac{u}{k(v)}\right)}, \prod_{v=0}^{t-1} \gamma(v)=\gamma(0) \gamma(1) \ldots \gamma(t-1),
$$

and $x(\tau(v))$ expresses the term $x(\tau(v) ; \varphi)$.

Since (1.1) denotes a realistic model of populations, we only consider the positive initial values $\varphi \in \mathscr{D}\left[-\tau_{0}, 0\right]$. Then, from (2.1) it follows that the solution $\{x(t ; \varphi)\}_{t \geq-\tau_{0}}$ of (1.1) is positive.

In addition, in view of the assumption (H2), we have

$$
u e^{r_{0}-\frac{r_{1} u}{k_{0}}} \leq f(v, u) \leq u e^{r_{1}-\frac{r_{0} u}{k_{1}}} \text { for } v \in \mathbb{Z}_{+} \text {and } u \geq 0,
$$

which results in

$$
f(v, u) \leq M=\frac{k_{1}}{r_{0}} e^{r_{1}-1} \text { for } v \in \mathbb{Z}_{+} \text {and } u \geq 0 .
$$

Now, for the positive initial value $\varphi \in \mathscr{D}\left[-\tau_{0}, 0\right]$, in combination with the assumption (H1), we have from (2.1) and (2.3) that

$$
\begin{aligned}
x(t ; \varphi) & \leq \gamma_{1}^{t} \varphi(0)+M \sum_{v=0}^{t-1} \gamma_{1}^{t-v-1} \\
& =\gamma_{1}^{t} \varphi(0)+M\left(1-\gamma_{1}\right)^{-1}\left(1-\gamma_{1}^{t}\right), t \in \mathbb{Z}_{+} .
\end{aligned}
$$

Hence, for $|\varphi| \in\left(0, \delta M\left(1-\gamma_{1}\right)^{-1}\right]$ with $\delta \geq 1$, coupled with (2.4), it holds that

$$
x(t ; \varphi) \leq \delta M\left(1-\gamma_{1}\right)^{-1}, t \in \mathbb{Z}_{+} .
$$

On the other hand, by the assumptions (H3)-(H4) and the left inequality in (2.2) we have

$$
f(v, u) \geq \Delta \text { for } v \in \mathbb{Z}_{+} \text {and } u \in\left[\Delta\left(1-\gamma_{0}\right)^{-1}, \delta M\left(1-\gamma_{1}\right)^{-1}\right],
$$

where the constant $\Delta$ defined as in (H4). Consequently, similar to (2.4) it follows from (2.1) and (2.6) that when $|\varphi| \in\left[\Delta\left(1-\gamma_{0}\right)^{-1}, \delta M\left(1-\gamma_{1}\right)^{-1}\right]$,

$$
\begin{aligned}
x(t ; \varphi) & \geq \gamma_{0}^{t} \varphi(0)+\Delta \sum_{v=0}^{t-1} \gamma_{0}^{t-v-1} \\
& =\gamma_{0}^{t} \varphi(0)+\Delta\left(1-\gamma_{0}\right)^{-1}\left(1-\gamma_{0}^{t}\right) \\
& \geq \Delta\left(1-\gamma_{0}\right)^{-1}, t \in \mathbb{Z}_{+} .
\end{aligned}
$$

Summarizing (2.5) and (2.7) we obtain the following result:

Theorem 1. Under the assumptions $(H 1)-H(4)$, the set $\left[\Delta\left(1-\gamma_{0}\right)^{-1}, \delta M(1-\right.$ $\left.\left.\gamma_{1}\right)^{-1}\right]$ is invariant relative to (1.1), here $\delta \geq 1$, and $\Delta$ is defined as in (H4). 
Let $\widetilde{m}=\min \left\{m, \frac{r_{0} k_{0}}{r_{1}}\right\}$. Note that in Theorem $1, \Delta=\widetilde{m}$ as $\delta=1$. Note further that, since $\gamma_{0}$ and $\gamma_{1} \in(0,1)$, for any given $\varepsilon>0$ and positive function $\varphi \in \mathscr{D}\left[-\tau_{0}, 0\right]$, there exists an $N(\varepsilon, \varphi)>0$ for which it follows that

$$
\gamma_{1}^{t}\left|\varphi(0)-M\left(1-\gamma_{1}\right)^{-1}\right|<\varepsilon, \gamma_{0}^{t}\left|\varphi(0)-\widetilde{m}\left(1-\gamma_{0}\right)^{-1}\right|<\varepsilon \text { for } t \geq N(\varepsilon, \varphi) .
$$

Consequently, from (2.4) and (2.7) we have

$$
x(t ; \varphi)<M\left(1-\gamma_{1}\right)^{-1}+\varepsilon \text { for } t \geq N(\varepsilon, \varphi)
$$

and

$$
x(t ; \varphi)>\widetilde{m}\left(1-\gamma_{0}\right)^{-1}-\varepsilon \text { for } t \geq N(\varepsilon, \varphi) .
$$

This induces our second result:

Theorem 2. Under the assumptions (H1)-(H3), the set $\left[\widetilde{m}\left(1-\gamma_{0}\right)^{-1}, M\left(1-\gamma_{1}\right)^{-1}\right]$ is a global attractor of (1.1), here $\widetilde{m}=\min \left\{m, \frac{r_{0} k_{0}}{r_{1}}\right\}$.

Next we continue to employ $\widetilde{m}=\min \left\{m, \frac{r_{0} k_{0}}{r_{1}}\right\}$ and consider the extreme stability with respect to (1.1). To do this, we first show the following conclusion.

Lemma 1. Suppose that $\{x(t ; \varphi)\}_{t \geq-\tau_{0}}(\{x(t)\}$ for short) is a solution of (1.1) and

$$
\frac{\widetilde{m}}{k_{1}\left(1-\gamma_{0}\right)}>1 \text {. }
$$

Then, under the assumptions (H1)-(H3), it follows that

$$
\begin{aligned}
& \limsup _{t \rightarrow \infty}\left|1-\frac{r(t) x(\tau(t))}{k(t)}\right| e^{r(t)\left(1-\frac{x(\tau(t))}{k(t)}\right)} \\
\leq & \max \left\{\left|1-\frac{r_{0} \widetilde{m}}{k_{1}\left(1-\gamma_{0}\right)}\right|,\left|1-\frac{r_{1} M}{k_{0}\left(1-\gamma_{1}\right)}\right|\right\} .
\end{aligned}
$$

Proof. We proceed in steps.

Assertion 1:

$$
\limsup _{t \rightarrow \infty}\left|1-\frac{r(t) x(\tau(t))}{k(t)}\right| \leq \max \left\{\left|1-\frac{r_{0} \widetilde{m}}{k_{1}\left(1-\gamma_{0}\right)}\right|,\left|1-\frac{r_{1} M}{k_{0}\left(1-\gamma_{1}\right)}\right|\right\} .
$$

Indeed, for a given solution $\{x(t ; \varphi)\}_{t \geq-\tau_{0}}$ of (1.1), Theorem 2 indicates that for any given $\varepsilon>0$, there exits an $N=N(\varepsilon, \varphi)$ such that

$$
\frac{\widetilde{m}}{1-\gamma_{0}}-\varepsilon<x(\tau(t))<\frac{M}{1-\gamma_{1}}+\varepsilon \text { for } t \geq N .
$$

Hence, from (2.10) we have for $t \geq N$,

$$
1-\frac{r(t) x(\tau(t))}{k(t)}<1-\frac{r_{0}}{k_{1}}\left(\frac{\widetilde{m}}{1-\gamma_{0}}-\varepsilon\right)
$$

and

$$
1-\frac{r(t) x(\tau(t))}{k(t)}>1-\frac{r_{1}}{k_{0}}\left(\frac{M}{1-\gamma_{1}}+\varepsilon\right) \text {. }
$$


Since $\varepsilon$ ia arbitrary, with the aid of (2.11)-(2.12), we see that our statement is true.

Assertion 2:

$$
\limsup _{t \rightarrow \infty} e^{r(t)\left(1-\frac{x(\tau(t))}{k(t)}\right)} \leq 1 .
$$

Indeed, by (2.8) and the left inequality in (2.10), we can derive

$$
\frac{x(\tau(t))}{k(t)}>\frac{\widetilde{m}}{k_{1}\left(1-\gamma_{0}\right)}-\frac{1}{k_{1}} \varepsilon>1-\frac{1}{k_{1}} \varepsilon \text { for } t \geq N,
$$

which lead to the result

$$
\liminf _{t \rightarrow \infty} \frac{x(\tau(t))}{k(t)} \geq 1
$$

This implies the assertion 2 is valid.

In summary, the conclusion (2.9) is effective. The proof is complete.

We now consider the extreme stability of (1.1).

Theorem 3. Let $\widetilde{m}=\min \left\{m, \frac{r_{0} k_{0}}{r_{1}}\right\}$. Suppose that condition (2.8) in Lemma 1 is verified and

$$
\max \left\{\left|1-\frac{r_{0} \widetilde{m}}{k_{1}\left(1-\gamma_{0}\right)}\right|,\left|1-\frac{r_{1} M}{k_{0}\left(1-\gamma_{1}\right)}\right|\right\}<1-\gamma_{1} .
$$

Then (1.1) is extremely stable under the assumptions (H1)-(H3).

Proof. By the condition (2.13), we can choose a real number $\widetilde{M}$ so that

$$
1-\gamma_{1}>\widetilde{M}>\max \left\{\left|1-\frac{r_{0} \widetilde{m}}{k_{1}\left(1-\gamma_{0}\right)}\right|,\left|1-\frac{r_{1} M}{k_{0}\left(1-\gamma_{1}\right)}\right|\right\} .
$$

Let $\{x(t)\}$ and $\{y(t)\}$ be any two positvie solutions of (1.1). Then, both $\{x(t)\}$ and $\{y(t)\}$ verify (1.1). Thereby, it follows that for any integer $N \geq 0$,

$$
x(t)=\prod_{v=N}^{t-1} \gamma(v) x(N)+\sum_{v=N}^{t-1}\left[\prod_{s=v}^{t-2} \gamma(s+1)\right] x(\tau(v)) e^{r(v)\left(1-\frac{x(\tau(v))}{k(v)}\right)}, t \geq N
$$

as well as

$$
y(t)=\prod_{v=N}^{t-1} \gamma(v) y(N)+\sum_{v=N}^{t-1}\left[\prod_{s=v}^{t-2} \gamma(s+1)\right] y(\tau(v)) e^{r(v)\left(1-\frac{y(\tau(v))}{k(v)}\right)}, t \geq N .
$$

Hence, it holds that

$$
\begin{aligned}
x(t)-y(t)= & \prod_{v=N}^{t-1} \gamma(v)(x(N)-y(N)) \\
& +\sum_{v=N}^{t-1}\left[\prod_{s=v}^{t-2} \gamma(s+1)\right]\left\{x(\tau(v)) e^{r(v)\left(1-\frac{x(\tau(v))}{k(v)}\right)}\right.
\end{aligned}
$$




$$
\left.-y(\tau(v)) e^{r(v)\left(1-\frac{y(\tau(v))}{k(v)}\right)}\right\}, t \geq N
$$

which derives

$$
\begin{gathered}
|x(t)-y(t)| \leq \gamma_{1}^{N-t}|(x(N)-y(N))| \\
+\sum_{v=N}^{t-1} \gamma_{1}^{t-v-1}\left|1-r(v) \frac{\xi(\tau(v))}{k(v)}\right| e^{r(v)\left(1-\frac{\xi(\tau(v))}{k(v)}\right)}|x(\tau(v))-y(\tau(v))|, t \geq N
\end{gathered}
$$

where $\xi(\tau(v))$ is between $x(\tau(v))$ and $y(\tau(v))$. Hence, Lemma 1 implies that there exists an $N$ for which

$$
\left|1-r(v) \frac{\xi(\tau(v))}{k(v)}\right| e^{r(v)\left(1-\frac{\xi(\tau(v))}{k(v)}\right)} \leq \widetilde{M} \text { for } t \geq N .
$$

Note that Theorem 1-2 imply that $\{x(t)-y(t)\}$ is bounded. Thus, we can set

$$
\limsup _{t \rightarrow \infty}|x(t)-y(t)|=w .
$$

Consequently, for any $\varepsilon>0$, there exists an $N_{1}=N_{1}(\varepsilon)$ large enough such that

$$
|x(\tau(t))-y(\tau(t))|<w+\varepsilon \text { for } t \geq N_{1} .
$$

For convenience, we deem that (2.16)-(2.17) hold whenever $t \geq N_{1}$. Then, by invoking (2.15)-(2.17) we have

$$
\begin{aligned}
|x(t)-y(t)| \leq & \gamma_{1}^{t-N_{1}}\left|\left(x\left(N_{1}\right)-y\left(N_{1}\right)\right)\right| \\
& +\frac{1-\gamma_{1}^{t-N_{1}}}{1-\gamma_{1}} \widetilde{M}(w+\varepsilon), t \geq N_{1},
\end{aligned}
$$

which results in

$$
w \leq \frac{1}{1-\gamma_{1}} \widetilde{M}(w+\varepsilon) .
$$

Now we impose (2.14) on the inequality above and then obtain $w=0$. This shows that $\lim _{t \rightarrow \infty}|x(t)-y(t)|=0$. The proof is complete.

Next we give an example to illustrate our results above.

\section{Example 1. Let}

$$
\gamma(t)=0.34+0.14 \sin t, r(t)=0.51+0.01 \sin t, k(t)=1.1+0.1 \sin t
$$

and consider

$$
x(t+1)=\gamma(t) x(t)+x(t-2) e^{r(t)\left(1-\frac{x(t-2)}{k(t)}\right)}, t \in \mathbb{Z}_{+} .
$$

Then

$$
\gamma_{0}=0.2, \gamma_{1}=0.48 ; r_{0}=0.5, r_{1}=0.52 ; k_{0}=1, k_{1}=1.2
$$


and, for $M=\frac{k_{1}}{r_{0}} e^{r_{1}-1}$ and $m=\frac{M}{1-\gamma_{1}} e^{r_{0}-r_{1} \frac{M}{k_{0}\left(1-\gamma_{1}\right)}}$,

$$
\widetilde{m}=\min \left\{m, \frac{r_{0} k_{0}}{r_{1}}\right\}=\frac{r_{0} k_{0}}{r_{1}}=\frac{50}{52}
$$

as well as

$$
1-\frac{r_{1}}{k_{0}} \cdot \frac{M}{1-\gamma_{1}}=-0.485080140,1-\frac{r_{0}}{k_{1}} \cdot \frac{\widetilde{m}}{1-\gamma_{0}}=0.4991987179 .
$$

Therefore, we have

$$
\frac{\widetilde{m}}{k_{1}\left(1-\gamma_{0}\right)}=1.001602564
$$

and

$$
\max \left\{\left|1-\frac{r_{0} \widetilde{m}}{k_{1}\left(1-\gamma_{0}\right)}\right|,\left|1-\frac{r_{1} M}{k_{0}\left(1-\gamma_{1}\right)}\right|\right\}<1-\gamma_{1} .
$$

So far, Theorem 2 implies that the interval $\left[\frac{125}{104}, \frac{60}{13} e^{-\frac{12}{25}}\right]$ is a global attractor of (2.18), and Theorem 3 shows that (2.18) is extremely stable. The following diagram supports partly our inferences.

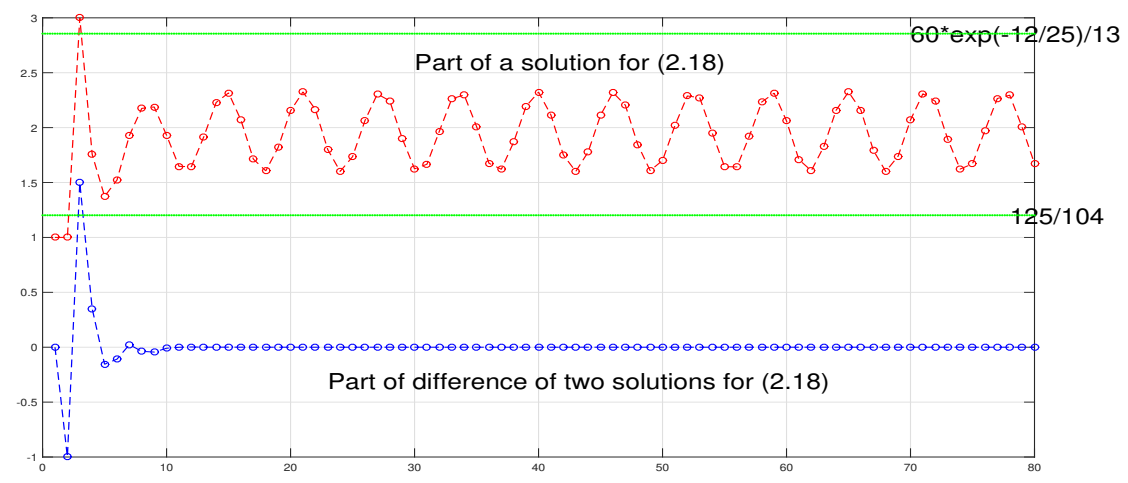

\section{Periodicity of (1.1)}

This section is devoted to consider the existence of periodic solutions of (1.1). For this purpose, we consider the Banach space

$$
l^{\infty}:=\left\{x=\{x(t)\}_{t \geq-\tau_{0}}: \sup _{t \geq-\tau_{0}}|x(t)|<\infty\right\}
$$

equipped with the norm defined by $\|x\|=\sup _{t \geq-\tau_{0}}|x(t)|$. Let

$$
\widetilde{m}=\min \left\{m, \frac{r_{0} k_{0}}{r_{1}}\right\}
$$


and the set $\delta \subset l^{\infty}$ be defined by

$$
\mathcal{s}:=\left\{\{x(t)\}_{t \geq-\tau_{0}} \in l^{\infty}: \widetilde{m}\left(1-\gamma_{0}\right)^{-1} \leq\|x\| \leq M\left(1-\gamma_{1}\right)^{-1}\right\} .
$$

The following result is similar to Lemma 1, and since the proof is analogous, we neglect it.

Lemma 2. Suppose that $\{x(t)\} \in \&$ and

$$
\frac{\widetilde{m}}{k_{1}\left(1-\gamma_{0}\right)} \geq 1
$$

Then, under the assumptions (H1)-(H2), it follows that

$$
\begin{aligned}
& \left|1-\frac{r(t) x(\tau(t))}{k(t)}\right| e^{r(t)\left(1-\frac{x(\tau(t))}{k(t)}\right)} \\
\leq & \max \left\{\left|1-\frac{r_{0} \widetilde{m}}{k_{1}\left(1-\gamma_{0}\right)}\right|,\left|1-\frac{r_{1} M}{k_{0}\left(1-\gamma_{1}\right)}\right|\right\}, t \geq 0 .
\end{aligned}
$$

We now establish a criterion to guarantee the existence of periodic solutions of (1.1). As we shall see below, our work is due to the celebrated Krasnoselskii fixedpoint theorem:

Lemma 3 ([1,4]). Let 8 be a closed, convex, and bounded subset of a Banach space $\mathcal{X}$ and $T, U: \delta \rightarrow \mathcal{X}$ be two operators such that

(i) $T$ is a contraction;

(ii) $U$ is completely continuous, and

(iii) $T x+U y \in \&$ for all $x, y \in \mathcal{8}$.

Then $T+U$ has a fixed point in 8 .

Theorem 4. Suppose that the assumptions (H1)-(H3) hold and $\{\gamma(t)\},\{r(t)\}$ and $\{k(t)\}$ are all periodic with the common period $\sigma$. Suppose further that

$$
\begin{gathered}
\tau(t+\sigma)=\tau(t)+\sigma \text { for all integer } t \geq 0, \\
\frac{\widetilde{m}}{k_{1}\left(1-\gamma_{0}\right)} \geq 1
\end{gathered}
$$

and

$$
\max \left\{\left|1-\frac{r_{0} \widetilde{m}}{k_{1}\left(1-\gamma_{0}\right)}\right|,\left|1-\frac{r_{1} M}{k_{0}\left(1-\gamma_{1}\right)}\right|\right\}<1-\gamma_{1} .
$$

Then the following is true:

(S1). equation (1.1) admits an unique $\sigma$-periodic solution;

(S2). any solution of (1.1) is asymptotically $\sigma$-periodic. 

by

Proof. To prove the first statement (S1), we introduce a subset $l_{a p}^{\infty} \subset l^{\infty}$ defined

$$
l_{a p}^{\infty}:=\left\{x \in l^{\infty}: x \text { is asymptotically } \sigma \text {-periodic }\right\} .
$$

Then $l_{a p}^{\infty}$ becomes a Banach space according to the norm $\|\cdot\|$ of $l^{\infty}$. Let

$$
\S_{a p}:=\left\{\{x(t)\}_{t \geq-\tau_{0}} \in l_{a p}^{\infty}: \widetilde{m}\left(1-\gamma_{0}\right)^{-1} \leq\|x\| \leq M\left(1-\gamma_{1}\right)^{-1}\right\} .
$$

It is clear that $\delta_{a p}$ is bounded, convex and closed. Now we define two operators $T, U: S_{a p} \rightarrow l_{a p}^{\infty}$ as follows:

$$
(T x)(t)= \begin{cases}0, & t=-\tau_{0},-\tau_{0}+1, \ldots, 0, \\ \gamma(t-1) x(t-1)+f(t-1, x(\tau(t-1))), & t \geq 1\end{cases}
$$

and

$$
(U x)(t)= \begin{cases}x(t), & t=-\tau_{0},-\tau_{0}+1, \ldots, 0, \\ 0, & t \geq 1,\end{cases}
$$

where $f(t-1, u)=u e^{r(t-1)\left(1-\frac{u}{k(t-1)}\right)}$. Then, for any $x, y \in \delta_{a p}$, we have from (H1) and (H3) that

$$
\begin{aligned}
(T x)(t)+(U y)(t) & =\gamma(t-1) x(t-1)+f(t-1, x(\tau(t-1))) \\
& \leq \gamma_{1} \frac{M}{1-\gamma_{1}}+M \\
& =M\left(1-\gamma_{1}\right)^{-1}, t \geq 1
\end{aligned}
$$

as well as

$$
\begin{aligned}
(T x)(t)+(U y)(t) & \geq \gamma_{0} \frac{\widetilde{m}}{1-\gamma_{0}}+\widetilde{m} \\
& =\widetilde{m}\left(1-\gamma_{0}\right)^{-1}, t \geq 1,
\end{aligned}
$$

where the relation $\widetilde{m}\left(1-\gamma_{0}\right)^{-1} \leq x(t-1) \leq M\left(1-\gamma_{1}\right)^{-1}$ have been employed for the first and second inequality. Hence, we have shown that

$$
\widetilde{m}\left(1-\gamma_{0}\right)^{-1} \leq(T x)(t)+(U y)(t) \leq M\left(1-\gamma_{1}\right)^{-1} \text { for all } t \geq-\tau_{0} .
$$

Next we proceed in several steps.

(i) Assertion 1: if $\{x(t)\}$ is asymptotically $\sigma$-periodic, then $\{f(t, x(\tau(t)))\}$ is also too.

Indeed, since $\{x(t)\}$ is asymptotically $\sigma$-periodic, we have the following decomposition

$$
x(t)=p(t)+q(t), t \geq-\tau_{0},
$$

where $\{p(t)\}$ is $\sigma$-periodic and $q(t) \rightarrow 0$ as $t \rightarrow \infty$. In addition, $f(t, u)=u e^{r(t)\left(1-\frac{u}{k(t)}\right)}$ implies that $\frac{\partial f(t, u)}{\partial u}=\left(1-\frac{r(t)}{k(t)} u\right) e^{r(t)\left(1-\frac{u}{k(t)}\right)}$. Then, by 
the mean value theorem, it holds that

$$
\begin{aligned}
|f(t, x(t))-f(t, p(t))| & =\left|x(t) e^{r(t)\left(1-\frac{x(t)}{k(t)}\right)}-p(t) e^{r(t)\left(1-\frac{p(t)}{k(t)}\right)}\right| \\
& =\left|1-\frac{r(t) \xi(t)}{k(t)}\right| e^{r(t)\left(1-\frac{\xi(t)}{k(t)}\right)}|x(t)-p(t)|,
\end{aligned}
$$

where, $\xi(t)$ is between $x(t)$ and $p(t)$.

Hence, in the light of (3.5)- (3.6) we see that $\{f(t, x(t))\}$ is asymptotically $\sigma$ periodic, which, together with (3.1), infers the assertion 1 is true.

(ii) Assertion 2: $T x+U y \in \oiint_{a p}$ for any $x, y \in \wp_{a p}$.

It is clear that the assertion 1, together with (3.4), results in $T x \in \mathcal{S}_{a p}$ for any $x \in \boldsymbol{S}_{a p}$. The belonging $U y \in \boldsymbol{S}_{a p}$ is obvious. Hence, with the aid of (3.4), we have shown that the assertion 2 is valid.

(iii) Assertion 3: $T$ is contractive.

Indeed, for any $x, y \in S_{a p}$ we have

$$
\begin{aligned}
(T x)(t)-(T y)(t) & =\gamma(t-1)(x(t-1)-y(t-1))+ \\
& (f(t-1, x(\tau(t-1)))-f(t-1, y(\tau(t-1)))), t \geq 1,
\end{aligned}
$$

which amounts to

$$
\begin{aligned}
& |(T x)(t)-(T y)(t)| \\
& \leq \gamma_{1}|| x-y||+ \\
& \left|1-\frac{r(t-1) \xi(\tau(t-1))}{k(t-1)}\right| e^{r(t-1)\left(1-\frac{\xi(\tau(t-1))}{k(t-1)}\right)}|| x-y||, t \geq 1,
\end{aligned}
$$

where $\xi(\tau(t-1))$ is between $x(\tau(t-1))$ and $y(\tau(t-1))$. Now by (3.3) we can choose a $\varepsilon_{0}>0$ so that

$$
\max \left\{\left|1-\frac{r_{0} \widetilde{m}}{k_{1}\left(1-\gamma_{0}\right)}\right|,\left|1-\frac{r_{1} M}{k_{0}\left(1-\gamma_{1}\right)}\right|\right\} \leq 1-\gamma_{1}-\varepsilon_{0} .
$$

Then, in association with (3.3) and Lemma 2, from (3.7) it holds that

$$
|(T x)(t)-(T y)(t)| \leq\left(1-\varepsilon_{0}\right)|| x-y \|, t \geq 1,
$$

which implies that $T$ is a contraction.

(iv) Assertion 4: $U$ is completely continuous.

As a matter of fact, $U \wp_{a p} \subset \mathbb{R}^{\tau_{0}}$ and the boundedness of $U \wp_{a p}$ mean that it is relatively compact. Consequently, $U$ is completely continuous.

In summary, the Krasnoselskii fixed-point theorem implies that, there exists $\widetilde{x} \in$ $\delta_{a p}$ so that

$$
\widetilde{x}(t)=(T \widetilde{x})(t)+(U \widetilde{x})(t), t \geq-\tau_{0},
$$

which indicates that

$$
\widetilde{x}(t+1)=\gamma(t) \widetilde{x}(t)+f(t, \widetilde{x}(\tau(t))), t \geq 0 .
$$


In other words, $\widetilde{x}$ is an asymptotically $\sigma$-periodic solution of (1.1).

(v) Assertion 5: Equation (1.1) has a unique $\sigma$-periodic solution.

Indeed, note that $\widetilde{x}=\{\widetilde{x}(t)\}$ can be decomposed by

$$
\widetilde{x}(t)=\widetilde{p}(t)+\widetilde{q}(t), t \geq-\tau_{0},
$$

where $\{\widetilde{p}(t)\}$ is $\sigma$-periodic, and $\widetilde{q}(t) \rightarrow 0$ as $t \rightarrow \infty$. Then, from (3.8) we have

$$
\widetilde{p}(t+1) \rightarrow \gamma(t) \widetilde{p}(t)+\widetilde{p}(\tau(t)) e^{r(t)(1-\widetilde{p(\tau(t))}} k \text { as } t \rightarrow \infty .
$$

Note that $\left\{\gamma(t) \widetilde{p}(t)+\widetilde{p}(\tau(t)) e^{r(t)\left(1-\frac{\widetilde{p}(\tau(t))}{k(t)}\right)}\right\}$ is $\sigma$-periodic. Hence, from (3.9) it follows that

$$
\widetilde{p}(t+1)=\gamma(t) \widetilde{p}(t)+\widetilde{p}(\tau(t)) e^{r(t)\left(1-\frac{\widetilde{p}(\tau(t))}{k(t)}\right)}, t \geq 0 .
$$

That is, $\{\widetilde{p}(t)\}$ is a $\sigma$-periodic solution of (1.1).

Let $\widetilde{y}$ be another $\sigma$-periodic solution of (1.1). Then Theorem 3 shows that

$$
\lim _{t \rightarrow \infty}|\widetilde{p}(t)-\widetilde{y}(t)|=0,
$$

which, induces

$$
\widetilde{y}(t)=\widetilde{p}(t) \text { for all } t \geq-\tau_{0} .
$$

That is, the first statement (S1) is true.

To prove the second statement (S2), assume that $\{x(t)\}$ is any solution of (1.1). Then, invoking Theorem 3 again, we arrive at

$$
\lim _{t \rightarrow \infty}|x(t)-\widetilde{p}(t)|=0
$$

which leads to

$$
x(t)=\widetilde{p}(t)+q(t), t \geq-\tau_{0},
$$

where $q(t) \rightarrow 0$ as $t \rightarrow \infty$. That is, $\{x(t)\}$ is an asymptotically $\sigma$-periodic solution of (1.1). The proof is complete.

Example 2. Let

$$
\gamma(t)=0.34+0.14 \sin t \pi, r(t)=0.51+0.01 \sin \frac{t \pi}{2}, k(t)=1.1+0.1 \sin \frac{t \pi}{2}
$$

and consider

$$
x(t+1)=\gamma(t) x(t)+x(\tau(t)) e^{r(t)\left(1-\frac{x(\tau(t))}{k(t)}\right)}, t \in \mathbb{Z}_{+},
$$

where

$$
\tau(t)=\left\{\begin{array}{l}
t-3, \quad t \text { is even; } \\
t-2, \quad \text { otherwise. }
\end{array}\right.
$$

Then, referring to example 1 we see that all the conditions in Theorem 4 are verified. Hence, (3.10) has a unique 4-periodic solution, and others are asymptotically 4-periodic. The facticity can be confirmed partly by the following: 


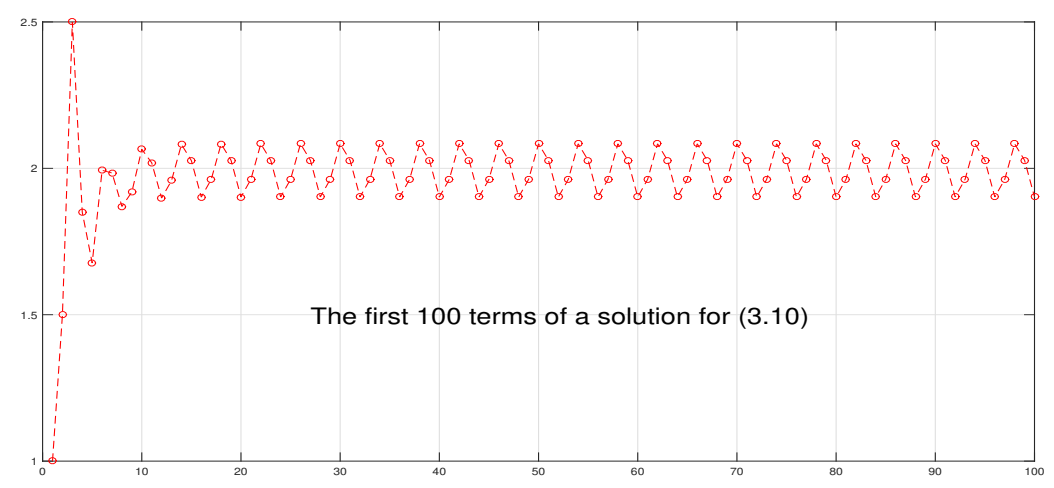

where we choose $x(-2)=1, x(-1)=1.5$ and $x(0)=2.5$ as the initial values of (3.10).

\section{ACKNOWLEDGEMENT}

The author is very grateful to the referees for their valuable suggestions.

Special thanks to the major research program of Colleges and Universities in Guangdong Province (No. 2017KZDXM054) for his support.

\section{REFERENCES}

[1] B. C. Dhage, "Remarks on two fixed-point theorems involving the sum and the product of two operators." Comput.Math. Appl., vol. 46, no. 12, pp. 1779-1785, 2003, doi: 10.1016/S08981221(03)00403-6.

[2] T. Diagana, "Almost automorphic solutions to a Beverton-Holt dynamic equation with survival rate." Appl. Math. Lett., vol. 36, no. 2, pp. 19-24, 2014, doi: 10.1016/j.aml.2014.04.011.

[3] Y. Hamaya, "Existence of almost periodic solutions of discrete ricker delay models." Int. J. Difference Equ., vol. 9, no. 2, pp. 187-205, 2014, doi: 10.1007/978-3-662-44140-4'9.

[4] M. A. Krasnoselskii, "Some problems of nonlinear analysis." Amer. Moth. Soc. Trans., vol. 10, no. 2, pp. 345-409, 1958, doi: 10.1090/trans2/010/13.

[5] E. Liz, "On the global stability of periodic Ricker maps." Electronic J. Qual. Theory of Diff. Equ., vol. 2016, no. 76, p. 8 PP., 2016, doi: 10.14232/ejqtde.2016.1.76.

[6] S. Mohamad and K. Gopalsamy, "Extreme stablity and almost periodicity in a discrete logistic equation." Tohoku Math. J., vol. 52, no. 1, pp. 107-125, 2000, doi: 10.2748/tmj/1178224661.

[7] J. Popenda and E. Schmeidel, "On the asymptotically periodic solution of some linear difference equations.” Arch. Math. (Brno), vol. 35, no. 1, pp. 13-19, 1999, doi: http://eudml.org/doc/248367.

[8] B. Ryals and R. J. Sacker, "Global stability in the 2D Ricker equation." J. Difference Equations Appl., vol. 21, no. 11, pp. 1068-1081, 2015, doi: 10.1080/10236198.2015.1065825.

[9] Z.-Q. Zhu and S. S. Cheng, "Stability analysis for multistep computational schemes." Comput.Math. Appl., vol. 55, no. 12, pp. 2753-2761, 2008, doi: 10.1016/j.camwa.2007.10.024. 
Author's address

Zhi-Qiang Zhu

Guangdong Polytechnic Normal University, Department of Information and Computing Science, 510665 Guangzhou, China

E-mail address: zzq@gpnu.edu.cn 